\title{
Detection of Fumonisins by ELISA Method
}

\author{
Ayumu Nagahara*, Satoshi Fukuda**, Mamoru Kikuchi** and \\ Susumu Kumagai***
}

\begin{abstract}
Abbreviation BSA, bovine serum albumin; OVA, ovalbumin; KLH, keyhole limpet hemocyanin ; i.p., intraperitoneal ; ELISA, enzyme-linked immunosorbent assay ; CI-ELISA, competitive indirect enzyme-linked immunosorbent assay ; CD-ELISA, competitive direct enzyme-linked immunosorbent assay ; $\mathrm{FB}_{1}$ (or $\mathrm{FB}_{2}$ ), fumonisin $\mathrm{B}_{1}$ (or $\mathrm{B}_{2}$ ) ; $\mathrm{PBS}$, phosphatebuffered saline.
\end{abstract}

Key words : fumonisin, ELISA, monoclonal antibody, corn

\section{Introduction}

Fumonisins are mycotoxins originally isolated from a culture of Fusarium moniliforme, one of the most commonly occurring fungi on $\operatorname{corn}^{1,2)}$. Fumonisin $\mathrm{B}_{1}$, which is the major fumonisin in both culture and naturally contaminated samples, has been shown to cause equine leukoenocephalomalacia ${ }^{3)}$ and porcine pulmonary edema ${ }^{4)}$, and to have cancerpromoting activity in rats $^{5)}$. Hepatocellular carcinoma was induced in rats by giving them fumonisin $\mathrm{B}_{1}$-containing feed ${ }^{6}$. Corresponding to the cancer-promoting and carcinogenic activities of the toxin, epidemiological studies have shown possible involvement of fumonisins in human esophagal cancer ${ }^{7}$. Analytical methods such as thin-layer chromatography (TLC) ${ }^{8,9)}$, gas chromatography-mass spectrometry (GC-MS) ${ }^{10}$, liquid secondary ion mass spectrometry (Liquid-SIMS) ${ }^{10)}$, and high-pressure liquid chromatography (HPLC) ${ }^{11,12)}$ have been used for the detection of fumonisins. These methods require extensive extraction, sample cleanup, or derivatization procedures. Therefore, these methods are not suitable for routine screening of large numbers of samples.

Immunoassays have recently been described as alternative methods for detection of fumonisins ${ }^{13,14)}$. In general, Low molecular weight compounds, such as fumonisins, have to be conjugated to carriers for immunization. Here we report the production of stable hybridomas by fusion of a mouse myeloma cell line, Sp-2, with mouse splenocytes immunized with fumonisin $\mathrm{B}_{1}$-ovalbumin conjugate $\left(\mathrm{FB}_{1}-\mathrm{OVA}\right)$ or fumonisin $\mathrm{B}_{1}$-keyhole limpet hemocyanin conjugate $\left(\mathrm{FB}_{1}-\mathrm{KLH}\right)$ together with Freund's complete and incomplete adjuvants, and the characterization of the anti-fumonisin monoclonal antibodies.

\footnotetext{
*Environmental Planning Department, Kikkoman Corporation, 350 Noda, Noda City, Chiba 2780037, Japan

${ }^{* *}$ Research and Development Division, Kikkoman Corporation, 399 Noda, Noda City, Chiba 2780037, Japan

*** Department of Biomedical Food Research, National Institute of Infectious Diseases, Toyama 1-231, Shinjuku-ku, Tokyo 162-8640, Japan
} 


\section{Methods}

Fumonisin $\mathrm{B}_{1}\left(\mathrm{FB}_{1}\right)$ was extracted from culuture of Fusarium moniliforme. $\mathrm{FB}_{1}$ was conjugated, using glutaraldehyde, with ovalbumin(OVA) and keyhole linpet hemocyanin $(\mathrm{KLH})$ for use as an immunogen $\left(\mathrm{FB}_{1}-\mathrm{OVA}\right.$ and $\mathrm{FB}_{1}-\mathrm{KLH}, \mathrm{FB}_{1} /$ protein ratio was $4: 5(\mathrm{w} / \mathrm{w}))^{15}$. $\quad \mathrm{FB}_{1}$ was also conjugated using m-maleimidobenzoyl- N-hydroxysuccinimide ester (MBS) to $\mathrm{BSA}\left(\mathrm{FB}_{1}-\mathrm{BSA} \text {, molar ratio of } \mathrm{FB}_{1} / \mathrm{BSA} \text { was } 70: 1\right)^{16}$.

Eight-week-old $\mathrm{BALB} / \mathrm{C}$ female mice were immunized by intraperitoneal (i.p.) injections, two with $\mathrm{FB}_{1}-\mathrm{OVA}$ and two with $\mathrm{FB}_{1}-\mathrm{KLH}(50 \mu \mathrm{g} /$ injection)with Freund's complete and incomplete adjuvants. At the final injection (30 days later), each high-titer mouse was given by i.p. injection of $100 / \mu$ of conjugate in PBS without adjuvant. Four days after the last injection, the mice were killed and spleens were removed aseptically and transferred to plastic Petri dishes containing Eagle's minimum essential medium (MEM). Myeloma cells (Sp-2) and spleen cells were fused with polyethylene glycol (PEG) ${ }^{17,18}$. The fused cells were suspended in hypoxanthine-aminopterin thymidine (HAT) medium containing thymocytes $\left(5 \times 10^{6} \mathrm{cells} / \mathrm{ml}\right)$ as feeder cells and pipetted into 96 -well culture plate. After HAT selection, supernatants from hybridoma cells were examined for the reactivity with $\mathrm{FB}_{1}$-BSA by ELISA and also measured for the specificity toward $\mathrm{FB}_{1}$ by competitive indivect ELISA(ClELISA). The same method of ELISA and CI-ELISA for antisera were used to the case of culture supernatants from hybridoma cells. After successive single-cell cloning by limiting dilution methods, five hybridoma cell lines producing anti- $\mathrm{FB}_{1}$ monoclonal antibodies were obtained. Four of the five hybridomas (OVA4A41, OVA4A48, OVA8D26 and OVA8D29) were obtained from the mouse irnmunized by $\mathrm{FB}_{1}-\mathrm{OVA}$ and one hybridoma (KLH17C10) was obtained from the mouse immunized by $\mathrm{FB}_{1}-\mathrm{KLH}$.

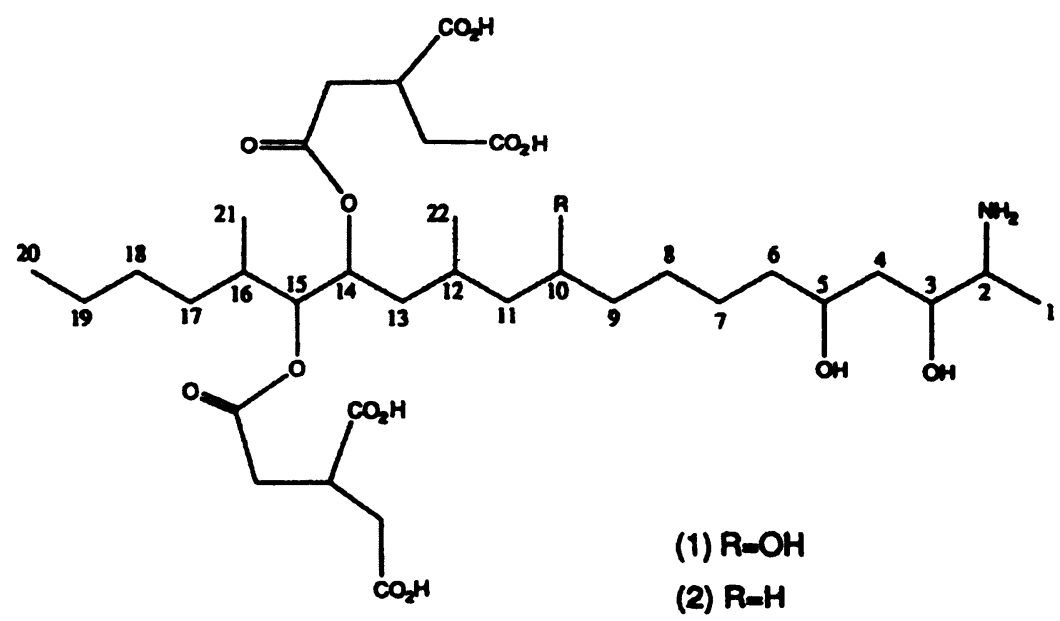

Fig. 1 Structure of Fumonisins.

(1) fumonisin $B_{1}$; (2) fumonisin $B_{2}$. 

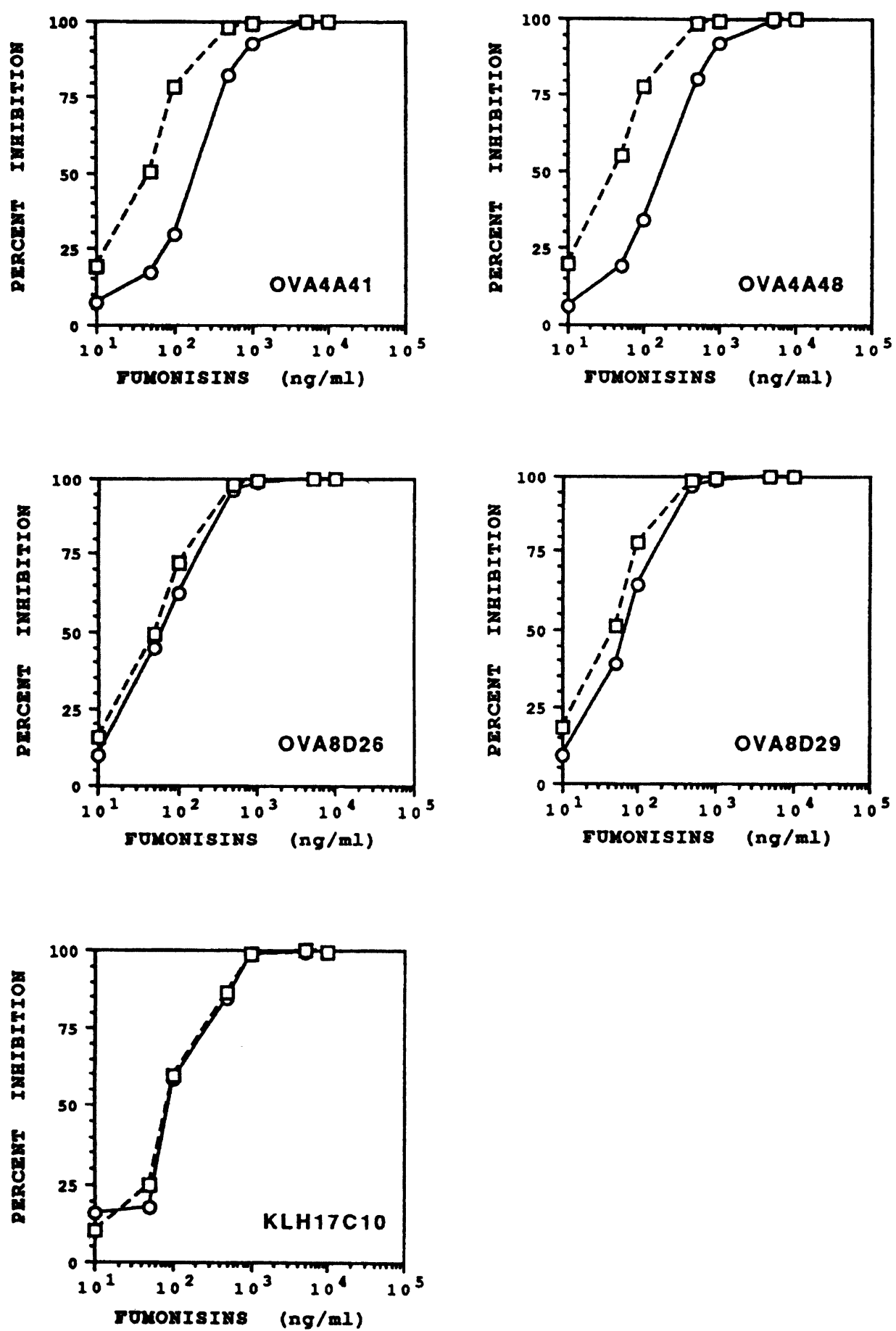

Fig. 2 Specificity of Anti-Fumonisin Monoclonal Antibodies produced by Seiected Hybridomas. Specificity was analyzed by CI-ELISA. Each data point represents the averagc vatue of triplicate measurements. $\bigcirc$, fumonisin $B_{1} ; \square$, fumonisin $B_{2}$. 
Table 1 Reactivity of Anti-Fumonisin Monoclonal Antibodies from Selected Hybridomas as measured by Cl-ELISA

\begin{tabular}{|c|c|c|c|}
\hline \multirow{2}{*}{ Hybridoma } & \multicolumn{2}{|c|}{$\begin{array}{l}\text { Amount required for } 50 \% \\
\text { inhibition }^{\mathrm{a}}(\mathrm{ng} / \mathrm{ml})\end{array}$} & \multirow{2}{*}{$\begin{array}{c}\text { Cross-reactivity } \\
(\%)\end{array}$} \\
\hline & Fumonisin $B_{1}$ & Pumonisin $\mathrm{B}_{2}$ & \\
\hline OVA4A41 & 255 & 49.5 & 520 \\
\hline OVA4A48 & 239 & 43.8 & 550 \\
\hline OVA8D26 & 65.3 & 50.9 & 130 \\
\hline OVA8D29 & 71.9 & 48.2 & 150 \\
\hline $\mathrm{KLH} 17 \mathrm{Cl} 10$ & 85.9 & 89.8 & 100 \\
\hline
\end{tabular}

${ }^{a}$ Data were estimated from Fig. 2.

${ }^{\mathrm{b}}$ Cross-reactivity defined as $\left(\mathrm{ng} / \mathrm{ml}\right.$ of fumonisin $\mathrm{B}_{1}$ required for $50 \%$ inhibition)/(ng/ $\mathrm{ml}$ of fumonisin $\mathrm{B}_{2}$ required for $50 \%$ inhibition) $\times 100$.

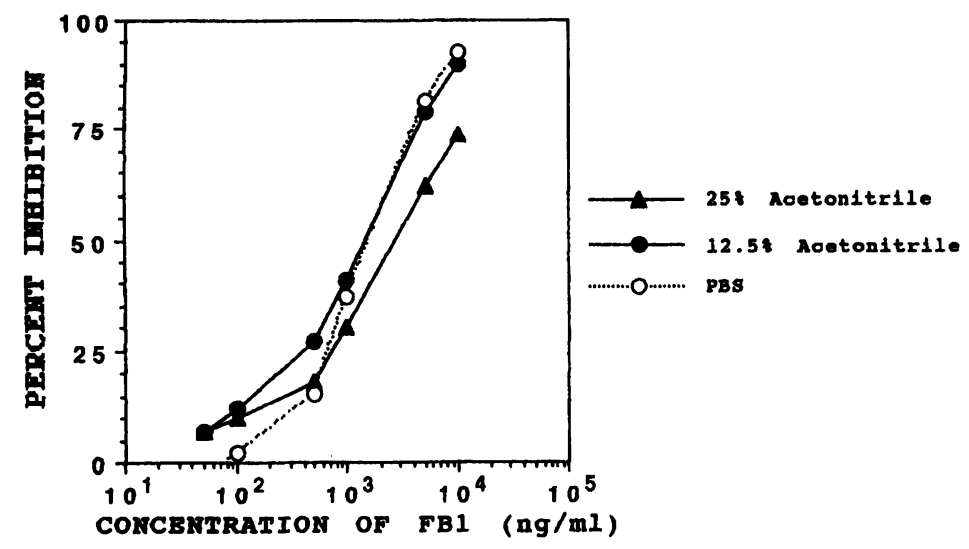

Fig. 3 Effects of acetonitrile concentration of solvent determined by CD-ELISA with monoclonal antibodies produced by $\mathrm{FB}_{1}$ - $\mathrm{BSA}$ conjugate.

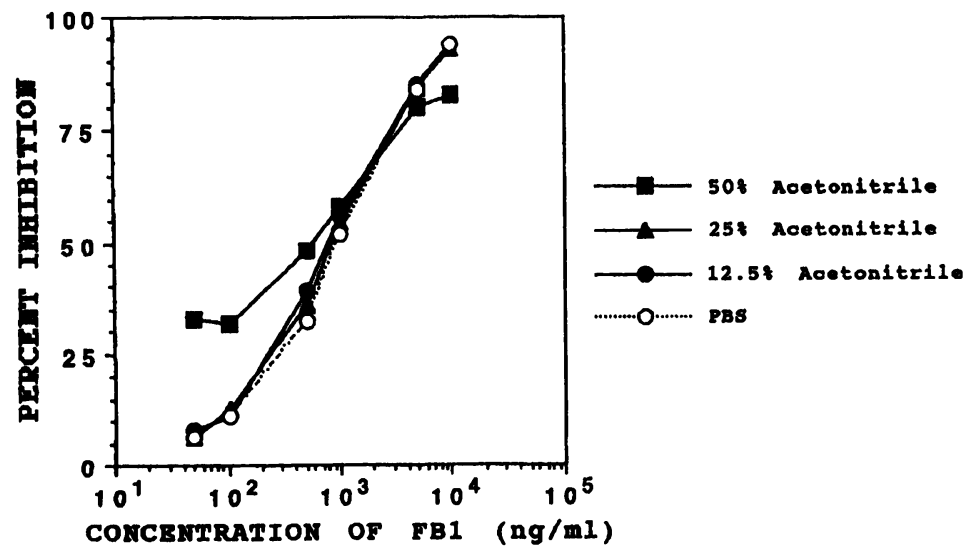

Fig. 4 Effects of acetonitrile concentration of solvent determined by CD-ELISA with monoclonal antibodies produced by $\mathrm{FB}_{1}-\mathrm{KLH}$ conjugate. 


\section{Results and Discussion}

The selected hybridoma cells were cultivated in Iscove's modified Dulbecco's medium
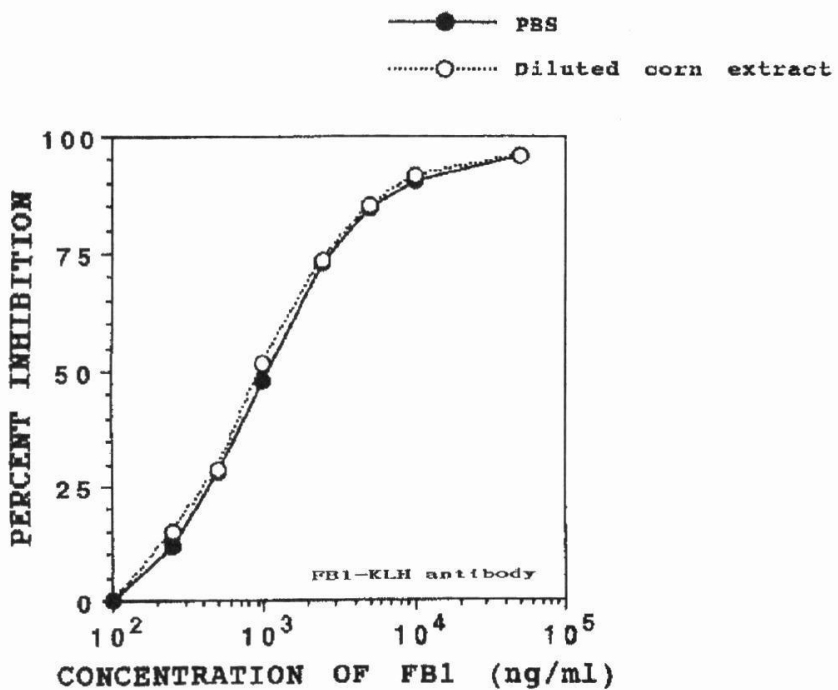

Fig. 5 Detection of $\mathrm{FB}_{1}$ by $\mathrm{CD}$-ELISA with monoclonal antibodies produced by $\mathrm{FB}_{1}$-KLH conjugate.

Contents of Food Checker for Fumonisin

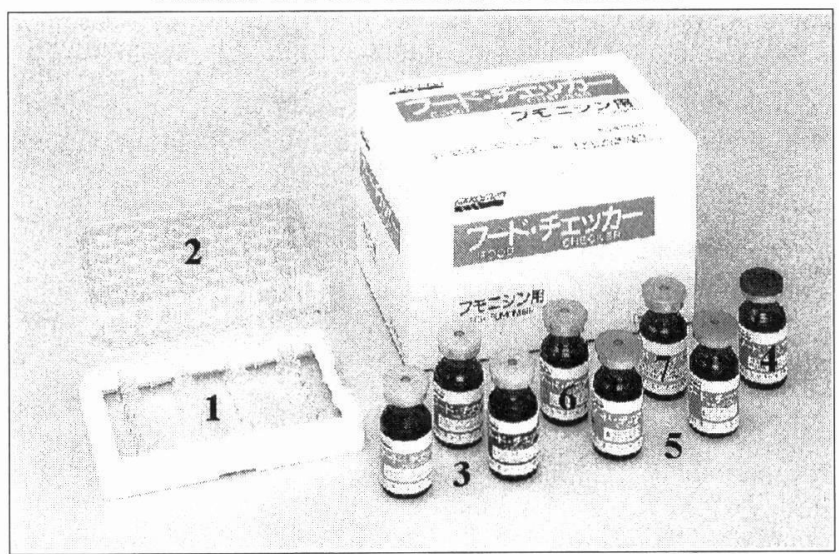

1. Antibody coated micro-well

2. 48 wells for concentrate

5. Substrate solutions

3. Positive control (Fumonisin $\mathrm{B}_{1}$ )

4. Enzyme conjugated Funnonisin
6. Stopping reagent

7. Washing solution(20x concentrated)

Photo Contents of FOOD CHECKER for FUMONISIN developed by KIKKOMAN CORPORATION. 
(IMDM) containing $20 \%$ fetal bovine serum (FBS). The specificities of antibodies were characterized by CI-ELISA with respect to $\mathrm{FB}_{1}, \mathrm{FB}$ and tricarballylic acid which is an analogue of the characteristic side-chains of fumonisins at position $\mathrm{C}_{14}$ and $\mathrm{C}_{15}$ (Fig. l). The subclass of secreted antibodies were identified with a mouse monoclonal antibody isotyping kit (Amersham, UK). The isotype of all monoclonal antibodies produced by five selected hybridomas were $\operatorname{IgG}_{1}$ with $\kappa$ light chain. Specificities of the antibodies are shown in Fig. 2 and Table. The concentration of $50 \%$ inhibition doses of fumonisin $\mathrm{B}_{1}$ and $\mathrm{B}_{2}$ for antibodies from five hybridoma clones ranged from 65 to $255 \mathrm{ng} / \mathrm{ml}$ for $\mathrm{FB}_{1}$ (mean, 144) and 44 to $86 \mathrm{ng} / \mathrm{ml}$ for $\mathrm{FB}_{2}$ (mean, 56). Reactivity was not observed with tricarballylic acid. Figure 2 shows the response curves of $\mathrm{FB}_{1}$. Response ranges of $\mathrm{FB}_{1}$ were from 10 to 1000 $-5000 \mathrm{ng} / \mathrm{ml}$. Cross-reactivity of our antibodies ranged from 100 to $550 \%$ for $\mathrm{FB}_{2}$ (mean, $290 \%$ ), however Azcona-Olivera et al. previously reported the value of $38 \%$ for the cross reactivity of $\mathrm{FB}_{2}$ measured by competitive direct ELISA ${ }^{14)}$. These results suggest that our high cross-reactive antibodies (produced by OVA4A41 and OVA4A48) may recognize a different region as antigenic determinant. The antibody secreted by clone KLH17C10 reacted almost to the same degree with $\mathrm{FB}_{1}$ and $\mathrm{FB}_{2}$. This property is desirable, since it enables simultaneous detection of both fumonisins.

This time, we used OVA and KLH as the carriers and also used BSA. FB FBSA $_{1}$ conjugate was also used for immunization and we got hybridomas from this (data not shown). Twenty five \% of acetonitrile effected to $\mathrm{FB}_{1}$-BSA antibodies but not effected to $\mathrm{FB}_{1}-\mathrm{KLH}$ antibodies by competitive direct ELISA(CD-ELISA) shown in Fig. 3 and 4 . Figure 5 indicate the CD-ELISA standard carve of $\mathrm{FB}_{1}$ in $\mathrm{PBS}$ and diluted corn extract with $\mathrm{FB}_{1}-\mathrm{KLH}$ antibody. Diluted corn extracts did not effect to the activity of $\mathrm{FB}_{1}-\mathrm{KLH}$ antibody.

We investigate the easy and rapid detection system for fumonisin using our $\mathrm{FB}_{1}-\mathrm{KLH}$ antibody. The name of this system is "FOOD CHECKER for FUMONISIN" shown in Photo. This ELISA system will be useful for detection of fumonisins in feeds and foods.

\section{References}

1) Bezuidenhout S. C., Gelderblom W. C. A., Gorst-Allman C. P., Horak R. M., Marasas W. F. O., Spiteller G. and Vleggaar R. (1988) Structure elucidation of the fumonisins, mycotoxins from Fusarium moniliforme, J. Cem. Soc., Chem. Commun., 743-745.

2) Sydenham E. W., Gelderblom W. C. A., Thiel P. G. and Marasas W. F. O. (1990) Evidence for the natural occurrence of fumonisin $B_{1}$, a mycotoxin produced by Fusarium moniliforme, in Corn, $J$. Agric. Food Chem., 38, 285-290.

3) Marasas W. F. O., Kellerman T. S., Gelderblom W. C. A., Coetzer J. A. W., Thiel P. G. and van der Jugt J. J. (1988) Leukoencephalomalacia in a horse induced by fumonisin $B_{1}$ isolated from Fusar. ium moniliforme, Onderstepoort J. Vet. Res., 55, 197-203.

4) Colvin B. M. and Harrison L. R. (1992) Fuminisin-induced pulmonary edema and hydrothorax in swine, Mycopathologia, 117, 79-82.

5) Gelderblom W. C. A., Jaskiewicz K., Marasas W. F. O., Thiel P. G., Horak R. M., Vleggaar R. and Kriek N. P. J. (1988) Fumonisins-Novel mycotoxins with cancer-promoting activity, Appl. Environ. Microbiol., 54, 1806-1811.

6) Gelderblom W. C. A., Kriek N. P. J., Marasas W. F. O. and Thiel P. G. (1991) Toxicity and carcinogenecity of the Fusarium moniliforme metabolite, fumonisin $\mathrm{B}_{1}$, in rats, Carcinogenesis, 12, 1247-1251.

7) Sydenham E. W., Gelderblom W. C. A., Thiel P. G. and Marasas F. O. (1990) Evidence for the natural occurrence of fumonisin $\mathrm{B}_{1}$, a mycotoxin produced by Fusarium moniliforme, in corn, $J$. 
Agric. Food Chem., 38, 285-290.

8) Rottinghaus G. E., Coatney C. E. and Minor H. C. (1992) A rapid, sensitive thin layer chromatography procedure for the detection of fumonisin $\mathrm{B}_{1}$ and $\mathrm{B}_{2}, J$. Vet. Diagn. Invest., 4, 326-329.

9) Cawood M. E., Gelderblom W. C. A., Vleggaar R., Behrend Y., Thiel P. G. and Marasas W. F. O. (1992) Isolation of the fumonisin mycotoxins: a quantitative approach, J. Agric. Food Chem., 39, 1958-1962.

10) Plattner R. D., Norred W. P., Bacon C. W., Voss K. A., Peterson R., Shackelford D. D. and Weisleder D. (1990) A method of detection of fumonisins in corn samples associated with field case of equine leukoencephalomalacia, Mycologia, 82, 698-702.

11) Shephard G. S., Sydenham E. W., Thiel P. G. and Gelderblom W. C. A. (1990) Quantitative determination of fumonisin $B_{1}$ and $B_{2}$ by high-performance liquid chromatography with fluorescence detection, J. Ljq. Chromatogr., 13, 2077-2087.

12) Scott P. M. and Lawrence G. A. (1992) Liquid chromatographic determination of fumonisins with 4-fluoro-7-nitrobenzofurazan, $J$ AOAC. Int., 75, 829-834.

13) Azcona-Olivera, J. I., Abouzied M. M., Plattner R. D., Norred W. P. and Pestka J. J. (1992) Generation of antibodies reactive with fumonisins $B_{1}, B_{2}$ and $B_{3}$ by using cholera toxin as the carrier-adjuvant, Appl. Environ. Microbiol., 58, 169-173.

14) Azcona-Olivera, J. I., Abouzied M. M., Plattner R. D. and Pestka J. J. (1992) Production of monoclonal antibodies to the mycotoxins fumonisins $\mathrm{B}_{1}, \mathrm{~B}_{2}$ and $\mathrm{B}_{3}$, J. Agric. Food Chem., 40, 531534.

15) Reichlin M. (1980) Use of glutaraldehyde as a coupling agent for proteins and peptide, In Methods ih Enzymology vol. 70 Immunochemical Techniques eds. van Vunakis H. and Langone J. L., Academic press, New York, pp. 159-165.

16) Miles L. E. and Hales C. N. (1968) Labelled antibodies and immunological assay systems, Nature, 219, 186-189.

17) Engvall E. and Perlmann P. (1972) Enzyme-linked immunosorbent assay, Elisa. 3. Quantitation of specific antibodies by enzyme-labeled anti-immunoglobulin in antigen-coated tubes, J. Immunol., 109, 129-135.

18) Kitagawa T., Fujitake T., Taniyama H. and Aikawa T. (1978) Enzyme immunoassay of viomycin. New cross-linking reagent for enzyme labeling and a preparation method for antiserum to viomycin, J. Biochem. (Tokyo), 83, 1493-1501.

19) Dixon D. E., Warner R. L., Ram B. P., Hart L. P. and Pastka J. J. (1987) Hybridoma cell line production of a specific monoclonal antibody to the mycotoxins zearalenone and $\boldsymbol{\alpha}$-zearalenol, $J$. Agric. Food Chem., 35, 122-126. 